\title{
Input of Innovative Technology for Surveillance and Improvement of Hand Hygiene: The Medihandtrace Contribution to Hand Disinfection Monitoring and Intervention
}

\author{
Jean-Charles Dufour ${ }^{1,2^{*}}$, Pauline Reynier ${ }^{1}$, Alberto Soto Aladro ${ }^{4}$ and Philippe Brouqui $3,4^{*}$ \\ ${ }^{1}$ Aix Marseille Université, UMR912 SESSTIM (AMU-Inserm-IRD-AMSE), Marseille, France \\ ${ }^{2}$ Assistance Publique des Hôpitaux de Marseille (APHM), BIOSTIC, Pôle de Santé Publique, Marseille, France \\ ${ }^{3}$ Aix Marseille Université, URMITE, UM63, CNRS 7278, IRD 198, Inserm 1095, France \\ ${ }^{4}$ Infectious Disease Unit CHU Nord, Institut Hospitalo-Universitaire Méditerranée Infection, France
}

*Corresponding authors: Philippe Brouqui, Aix Marseille Université, URMITE, UM63, CNRS 7278, IRD 198, Inserm 1095, France, Tel: +33-4-91-39-65-00; E-mail: philippe.brouqui@univ-amu.fr

Jean-Charles Dufour, Aix Marseille Université, UMR912 SESSTIM (AMU-Inserm-IRD-AMSE), Marseille, France, Tel: +33-4-91-39-65-00; Email: Jeancharles.DUFOUR@ap-hm.fr

Received date: July 23, 2015; Accepted date: August 13, 2015; Published date: August 20, 2015

Copyright: (c) 2015 Dufour JC, et al. This is an open-access article distributed under the terms of the Creative Commons Attribution License, which permits unrestricted use, distribution, and reproduction in any medium, provided the original author and source are credited.

\section{Introduction}

Hand hygiene practice has been proven efficient to limit the spreading of infectious diseases in hospital setting [1]. Measurement of hand hygiene compliance is an important component of infection control programs $[2,3]$. The WHO recommends regular monitoring to improve the hand hygiene compliance. Currently, most healthcare facilities measure hand hygiene compliance almost exclusively via direct human observation of healthcare workers (HCW) [4,5]. While considered as the "gold standard" [6], direct observation is laborintensive and susceptible to observer biases [6]. The observation bias has been investigated and has proven to influence the behavior of the observed person [7]. Furthermore, the reliability of hand hygiene audits based on direct observation as a reflection of overall performance can be adversely affected by sporadic or inconsistent sampling [6]. Using such monitoring methods, the compliance of hand hygiene varies from $4 \%-100 \%$. Despite many interventions, hand hygiene practices remain poorly followed in most hospitals.

In order to maintain and/or improve hand disinfection (HD), in an interventional research framework or in routine care, one must: 1) be able to assess objectively, and ideally individually and in a continuous manner, hand hygiene; 2) identify the determinants on which it is possible to act globally and/or individually; 3 ) implement measures that will act on these determinants; 4) evaluate the effect of these actions.

Within the MediHandTrace project, our objective was to setup, and improves step by step, a system based on radio frequency identification (RFID) technology, integrated in the HCWs workflow, in order to generate the data necessary to points 1,2 and 4 and implement some solutions responding to point 3 .

Our objective in this paper, is not to report results of the data analysis which are in process and valuable but limited to our care unit, but to report the MediHandTrace core functionalities and also discuss the new paradigm, capacities and ideas that can emerge when such innovative technology is routinely used and massive data are at disposal. That is precisely the context in which more and more care processes will be conducted.

\section{Materials and Methods}

MediHandTrace (patent ${ }^{\circ}$ FR 12/60453) system has been set up by a group consisting of HCWs, academic researchers and industrialists. It has been used in every day practice since September 2013 within the Infectious Disease Unit at the 'Hôpital Nord' of Marseille [8].

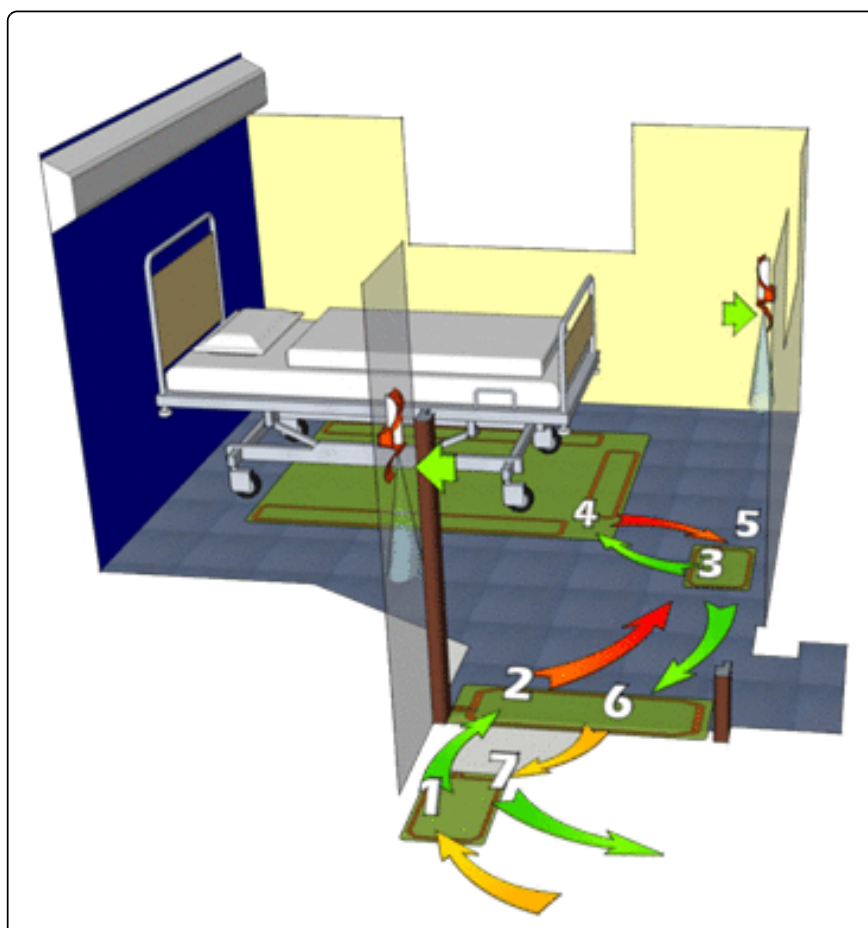

Figure 1: MediHandTrace ground antennas and HASD inside and outside a patient room with numbered signal recorded. Yellow and orange arrows indicated the pathway of potentially contaminated HWC, green arrows indicating a safe pathway after hand disinfection. The optimal pathway for a safe care is to pass through point 1-2-3-4-5-6 and 7. This figure is an adaptation of figure 1 in reference [8]. 
Citation: Dufour JC, Reynier P, Aladro AS, Brouqui P (2015) Input of Innovative Technology for Surveillance and Improvement of Hand Hygiene: The Medihandtrace Contribution to Hand Disinfection Monitoring and Intervention. Clin Microbiol 4: 216. doi: $10.4172 / 2327-5073.1000216$

Page 2 of 4

The system is based on passive RFID technology in order to record HCWs paths and their use of hydroalcoholic solution dispensers (HASD) fixed outside and inside patient rooms (HASD integrate microswitches detecting the taken dose). Each room is equipped with four ground antennas (under each HASD, on the doorstep and around the patient's bed). HCWs wear shoe-inserted RFID tag (all HCWs have given their explicit consent for this identification) (Figure 1). Tagged shoes could be considered as an ID badge and be mandatorily worn. All rooms are connected to a dedicated application on an intranet secured server where data are centralized and analyzed. The core concept of MediHandTrace is that a very elementary binary signal (HCWs' shoe-inserted RFID tags is detected or not by antennas situated at key locations) can be used, when temporally chained with other signals so as to precisely calculate the path of a HCW and record when he/she uses or not the HASD at disposal [8]. This application gives HCWs hand disinfection results in real-time. Initially conceived as an auditing tool, the database of this application has been used to explore many solutions that can positively impact compliance with hand hygiene. A basic program held by the machine is able to give in real time basic statistics such as personal, worker category, and total staff compliance to hand disinfection or the volume alcohol solution used by room by date. More sophisticated statistics (see variables recorded below) can be extracted from the data base and these statistics are available on demand. each path of HCWs and especially to categorize these paths according to which and when HASD is used. A path is defined as a sequence of HCW RIFD tag detection on antenna locations beginning and ending by usage of the outside HASD (or by the doorstep antenna detection if the HASD is not used). Several explorations of HD can be performed using this notion of HCW path. For example, we decided to categorize and study 3 path classes: usage of the bedside HASD just before contact with patient, usage of the outside HASD before contact with patient, no usage of HASD before contact with patient.

The system is also valuable to generate a set of variables which strongly enrich our knowledge of HCW paths and which could not be so easily and continuously collected without such technology. Thus, we can automatically calculate the duration of paths, the number of HCW simultaneously present in the patient' bedroom, the time laps between two paths, the number of times (and time-length) when a HCW stays near the bed, and so on. In addition to these automatically calculated variables, we can link paths with complementary variables such as: patient data; room architecture and disposition; HCW characteristics. So far we have considered 5 main categories of information which cover more than fifty elementary variables linked to each HCW path (Table 1). From September 2013 to March 2015 (18 months) our database has grown to a collection of 48000 paths which represent almost 2.5 million of data values.

\section{Results}

By using binary RFID time stamped signals generated by the system, it has been possible to record automatically and in real time

\begin{tabular}{|c|c|}
\hline Path variables & Patient data \\
\hline Path duration & Sex \\
\hline Period of the day/week & Age \\
\hline Number of HCW in the room & ICD10* pathology code \\
\hline Move over the patient's bed & Duration of the stay \\
\hline Laps between paths & Room information \\
\hline HCW characteristics & Position of the HASD \\
\hline Sex & Position of the bed \\
\hline Age & Size of the room (square meters) \\
\hline Hand usage (left/right) & HD variables \\
\hline Care specialty/function & Usage of the inside-room DHSA \\
\hline Job tenure & Usage of the outside-room DHSA \\
\hline *ICD10: 10th revision of the International Statistical Classification of Diseases and related health problems & \\
\hline
\end{tabular}

Table 1: The 5 main categories and some types of variables collected within MHT.

Beside the HD, data from MediHandTrace can be used to observe and follow over time other care-related information as for example global activity of the unit and to visualize the variation of work load during the day (Figure 2). 
Citation: Dufour JC, Reynier P, Aladro AS, Brouqui P (2015) Input of Innovative Technology for Surveillance and Improvement of Hand Hygiene: The Medihandtrace Contribution to Hand Disinfection Monitoring and Intervention. Clin Microbiol 4: 216. doi: $10.4172 / 2327-5073.1000216$

Page 3 of 4

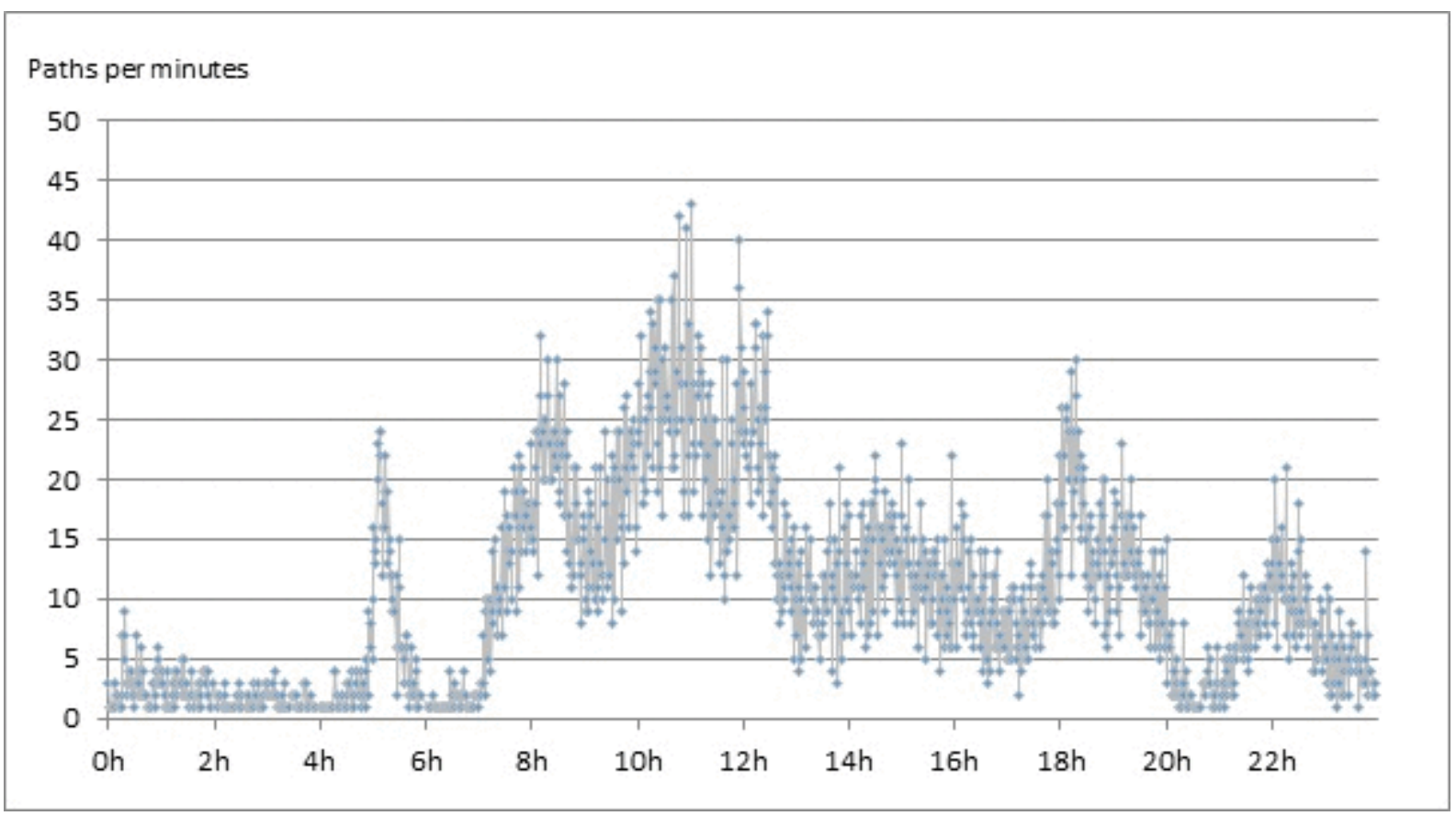

Figure 2: Health care mean work flow in the 7 rooms as represented by the number of path per minute, indexed on a daily basis and obtained from 48000 paths collected during the study. This reflects perfectly the work flow; 5:30 AM is night nurses last round, $7 \mathrm{AM}$ is breakfast time, followed by nursing and medical round until $1 \mathrm{PM}$, etc.

MediHandTrace, is also a tool which allows impact evaluation of specific interventions on HD. These interventions can be directly integrated and extend the initial system: we are currently experimenting 1) real-time sound alarms from the HASD if HD hasn't be done and 2) SMS weekly sent by the system to the HCWs in order to give information about their progression concerning their paths with and without HD. Interventions can also be external to the system and concern organizational measures: for example we have planned to observe the impact of the nurse care kits usage for the hygiene of bedridden patients (impact of HD but also on multiple entrances/exits, care duration, etc.). Healthcare associated infection (HCAI) is affecting approximately 5 million people each year in Europe with an estimated cost of 13 billion- 24 billion $€$. In the USA, the annual rate of HCAI was estimated 1.7 million cases, with an economic impact of approximately 6.5 billion US\$. A reduction of $10 \%$ of HCAI would save enough life and money to equip most healthcare center. The goal is not to equip all health care units of a hospital but those in which the number of HAI is highest such as ICU. Some mobile devices are also available for temporarily survey by infection control teams in replacement of WHO survey of hand disinfection monitoring.

\section{Discussion}

IT systems are increasingly used for their ability to automate and facilitate the collection of data both in controlled research context and in real condition. Technologies can now be worn by people and/or embedded in their environment and can interact with them. Thus they are particularly suitable for research in which, beyond the observation, an interventional action is desired. MediHandTrace is an example of the integration of an innovative technology both for interventional research and useful for daily practices observation concerning HD and other care habits related to HCW paths.

The massive, individual, systematic and continuous data collection is a new paradigm where several skills are required not only for data management and analysis but also because "big data" enrich the original question and give it different angles of analysis. In our case, concerning the HD: ethical, sociological, industrial, architectural, organizational considerations and possible responses have emerged from data at our disposal. Not only was multidisciplinary work consistent with "big data", it was also required from the outset regarding the studied problem: there are many heterogeneous factors that can be envisioned to explain HD practices, such as HCW behavior, HCW knowledge about contagious risks, the relative location of HASD and patient bed, the HCW workflow, the period of the day, patient condition and maybe other factors which have to be identified. MediHandTrace is a RFID-based tracking system allows exhaustive recording of HCW paths and many variables related to these paths. The system is well adapted to investigate extensively and act on well-known HD compliance factors as well as to understand better unstudied or yet to be discovered factors, thanks to the power of the exhaustive collection of paths it records.

\section{References}

1. Marra AR, Edmond MB (2014) New technologies to monitor healthcare worker hand hygiene. Clin Microbiol Infect 20: 29-33.

2. Boyce JM, Pittet D; Healthcare Infection Control Practices Advisory Committee Society for Healthcare Epidemiology of America. Association for Professionals in Infection Control. Infectious Diseases Society of America. Hand Hygiene Task Force (2002) Guideline for Hand Hygiene in Health-Care Settings: recommendations of the Healthcare Infection 
Citation: Dufour JC, Reynier P, Aladro AS, Brouqui P (2015) Input of Innovative Technology for Surveillance and Improvement of Hand Hygiene: The Medihandtrace Contribution to Hand Disinfection Monitoring and Intervention. Clin Microbiol 4: 216. doi: $10.4172 / 2327-5073.1000216$

Page 4 of 4

Control Practices Advisory Committee and the HICPAC/SHEA/APIC/ IDSA Hand Hygiene Task Force. Infect Control Hosp Epidemiol 23: S3-40.

3. Pittet D, Allegranzi B, Boyce J; World Health Organization World Alliance for Patient Safety First Global Patient Safety Challenge Core Group of Experts (2009) The World Health Organization Guidelines on Hand Hygiene in Health Care and their consensus recommendations. Infect Control Hosp Epidemiol 30: 611-622.

4. Sax H, Allegranzi B, Uçkay I, Larson E, Boyce J, et al. (2007) 'My five moments for hand hygiene': a user-centred design approach to understand, train, monitor and report hand hygiene. J Hosp Infect 67: 9-21.

5. Erasmus V, Daha TJ, Brug H, Richardus JH, Behrendt MD, et al. (2010) Systematic review of studies on compliance with hand hygiene guidelines in hospital care. Infect Control Hosp Epidemiol 31: 283-294.
6. Haas JP, Larson EL (2007) Measurement of compliance with hand hygiene. J Hosp Infect 66: 6-14.

7. Haessler S (2014) The Hawthorne effect in measurements of hand hygiene compliance: a definite problem, but also an opportunity. BMJ Qual Saf 23: 965-967.

8. Boudjema S, Dufour JC, Aladro AS, Desquerres I, Brouqui P (2014) MediHandTrace ${ }^{\bullet}$ : a tool for measuring and understanding hand hygiene adherence. Clin Microbiol Infect 20: 22-28. 